\title{
Charge-Induced Fluctuation Forces in Graphitic Nanostructures
}

\author{
D. Drosdoff, ${ }^{1,2}$ Igor V. Bondarev, ${ }^{2}$ Allan Widom, ${ }^{3}$ Rudolf Podgornik,,${ }^{4,5}$ and Lilia M. Woods ${ }^{1}$ \\ ${ }^{1}$ Department of Physics, University of South Florida, Tampa, Florida 33620, USA \\ ${ }^{2}$ Department of Mathematics and Physics, North Carolina Central University, \\ Durham, North Carolina 27707, USA \\ ${ }^{3}$ Department of Physics, Northeastern University, Boston, Massachusetts 02115, USA \\ ${ }^{4}$ Department of Theoretical Physics, Jozef Stefan Institute, SI-1000 Ljubljana, Slovenia \\ ${ }^{5}$ Department of Physics, University of Massachusetts, Amherst, Massachusetts 01003, USA \\ (Received 9 February 2015; revised manuscript received 25 June 2015; published 21 January 2016)
}

\begin{abstract}
Charge fluctuations in nanocircuits with capacitor components are shown to give rise to a novel type of long-ranged interaction, which coexist with the regular Casimir-van der Waals force. The developed theory distinguishes between thermal and quantum mechanical effects, and it is applied to capacitors involving graphene nanostructures. The charge fluctuations mechanism is captured via the capacitance of the system with geometrical and quantum mechanical components. The dependence on the distance separation, temperature, size, and response properties of the system shows that this type of force can have a comparable and even dominant effect to the Casimir interaction. Our results strongly indicate that fluctuation-induced interactions due to various thermodynamic quantities can have important thermal and quantum mechanical contributions at the microscale and the nanoscale.
\end{abstract}

DOI: 10.1103/PhysRevX.6.011004

Subject Areas: Condensed Matter Physics, Graphene, Interdisciplinary Physics

\section{INTRODUCTION}

Fluctuation-induced interactions have widespread applications in materials and microscaled and nanoscaled devices. The much-studied Casimir and van der Waals interactions are due to electromagnetic mode fluctuations captured via the dielectric and magnetic response properties of the objects [1,2]. Such forces are universal, and they are especially prominent at micron distances and below. Fluctuations of many other observables are also possible, which may give rise to different interactions [3]. In particular, voltage fluctuations in capacitor systems [4] and wires $[5,6]$ have been of much interest, especially for the operation of devices [7]. Charge fluctuations and the induced forces are of relevance to biological and chemical matter. Specifically, it has been shown that thermal charge fluctuations in ionic solutions can generate an attractive long-ranged dispersive force even between molecules charged with the same sign $[8,9]$. Fluctuations originating from charge disorder on neutral slabs have been shown to give an additional contribution to the net interacting force while completely masking the typical Casimir-van der Waals interaction [10].

The isolation of single graphene layers and synthesis of related nanostructures, such as carbon nanotubes and

Published by the American Physical Society under the terms of the Creative Commons Attribution 3.0 License. Further distribution of this work must maintain attribution to the author $(s)$ and the published article's title, journal citation, and DOI. graphene nanoribbons (GNRs), have brought new directions in electromagnetic fluctuation-induced phenomena. For example, the Casimir-van der Waals force involving graphene systems has nontrivial scaling behavior, which can be tuned via temperature, chemical potential, and doping modifications [11-15]. Understanding the electromagnetic fluctuations in the context of building a fundamental knowledge and making technological designs for such materials can hardly be overestimated.

Capacitor systems involving graphitic nanostructures have also been studied extensively. The application of a bias on a graphene system above a substrate is of great relevance to many quantum devices. A key factor has been the control of the local graphene electrochemical potential for the device functionality. Capacitance measurements can also be utilized as means to probe the basic electronic properties of graphitic nanostructures and to learn about their Dirac-like nature. In particular, the 2D character of graphene is exhibited in the quantum capacitance [16,17], a concept related to the partially screened electrostatic field due to the atomic scale of the graphene thickness [18]. Despite the presence of charges and voltage bias in graphene-based capacitive systems, their fluctuations and subsequent induced interaction effects have never been considered.

In this paper, we investigate fluctuating charges transferred through the connection of a wire in a capacitor system. Such charge-induced fluctuations are governed by fluctuation-dissipation relations giving rise to a novel longranged interaction of Casimir-like nature. The fundamental 
difference with the typical Casimir force lies in their origin. While the Casimir phenomenon is due to the electromagnetic fluctuation excitations associated with the dielectric and magnetic response of each plate, the charge-induced effect is due to monopolar charge fluctuations between the plates transferred through the wire. Since in many cases nanostructures are characterized by a reduced Casimir force as compared to 3D [19-22], nanocapacitors offer the possibility of finding regimes where the charge-induced fluctuation interaction can be dominant.

We present a general theory utilizing the capacitance concept and distinguishing between thermal and quantum mechanical effects through characteristic dependences on distance, temperature, and other factors. Graphene-based systems are taken as templates for which this theory is applied. Comparison with the typical Casimir interaction helps us understand various regimes where each type of interaction may be more important.

\section{CHARGE FLUCTUATION INTERACTIONS AND CAPACITANCE}

The capacitor system under consideration is shown in Fig. 1. It consists of two plates connected by a wire. The voltage fluctuations of the wire induce excess fluctuating charges on the capacitor. As a result, a force originating from these types of fluctuations is induced between the plates. It is clear that this monopolar charge fluctuations force differs in origin from the dipolar fluctuation force giving rise to the well-established van der Waals-Casimir effect. The description of the interaction is closely related to the surface charge density response $\delta \sigma$ due to the external voltage $\delta V$ via the interaction Hamiltonian $\delta H=-\int d^{2} r^{\prime} \sigma\left(\mathbf{r}^{\prime}, t\right) \delta V\left(\mathbf{r}^{\prime}, t\right):$

$$
\delta \sigma(\mathbf{r}, t)=\int_{-\infty}^{t} d t \int d^{2} r^{\prime} C\left(\mathbf{r}, \mathbf{r}^{\prime} ; t-t^{\prime}\right) \delta V\left(\mathbf{r}^{\prime}, t^{\prime}\right)
$$

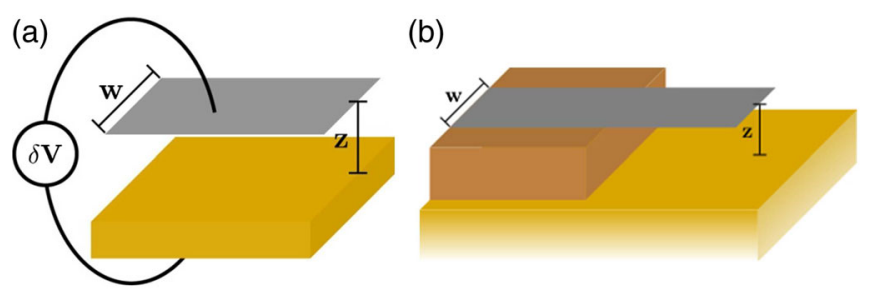

FIG. 1. (a) Ideal system under consideration. A parallel plate capacitor connected by a wire supporting voltage fluctuations $\delta V$. One of the plates can be a graphene ribbon with a width $w$ above a thick metallic substrate separated by a distance $z$. The capacitor system is assumed to be in an environment of air with $\epsilon \approx 1$. (b) Experimentally realistic setting. The wire is represented by a metallic connection between the "bottom" metal plate and the "top" graphene nanoribbon.
A key quantity here is the capacitance of the system, which can be written in terms of the commutator of the charge density-density correlations as [23]

$$
C\left(\mathbf{r}, \mathbf{r}^{\prime} ; t-t^{\prime}\right)=\frac{i}{\hbar}\left\langle\left[\sigma(\mathbf{r}, t), \sigma\left(\mathbf{r}^{\prime}, t^{\prime}\right)\right]\right\rangle,
$$

where $\langle\cdots\rangle=\operatorname{Tr}(\rho \ldots)$, with $\rho$ being the unperturbed density matrix.

The density correlation can further be represented as

$$
\begin{gathered}
\frac{1}{2}\left\langle\delta \sigma(\mathbf{r}, t) \delta \sigma\left(\mathbf{r}^{\prime}, t^{\prime}\right)+\delta \sigma\left(\mathbf{r}^{\prime}, t^{\prime}\right) \delta \sigma(\mathbf{r}, t)\right\rangle \\
=\int \frac{d \omega}{2 \pi} \bar{S}\left(\mathbf{r}, \mathbf{r}^{\prime} ; \omega\right) e^{-i \omega\left(t-t^{\prime}\right)},
\end{gathered}
$$

where the structure factor $\bar{S}\left(\mathbf{r}, \mathbf{r}^{\prime} ; \omega\right)$ is related to the frequency-dependent capacitance via the fluctuation-dissipation theorem as $\bar{S}\left(\mathbf{r}, \mathbf{r}^{\prime} ; \omega\right)=$ $\hbar \operatorname{Im} C\left(\mathbf{r}, \mathbf{r}^{\prime} ; \omega\right) \operatorname{coth}\left(\hbar \omega / 2 k_{B} T\right)$. Using the fluctuationdissipation theorem in Eq. (3) and the identity $\operatorname{coth}(x)=\sum_{n=-\infty}^{\infty} x /\left(x^{2}+n^{2} \pi^{2}\right)$, one obtains for the equal time charge density correlations

$\int \frac{d \omega}{2 \pi} \bar{S}\left(\mathbf{r}, \mathbf{r}^{\prime} ; \omega\right)=k_{B} T \sum_{n=-\infty}^{\infty} \int \frac{d \omega}{\pi} \frac{\omega \operatorname{Im} C\left(\mathbf{r}, \mathbf{r}^{\prime} ; \omega\right)}{\omega^{2}+\omega_{n}^{2}}$,

with $\omega_{n}=2 \pi n k_{B} T / \hbar$ being the Matsubara frequencies. From the theory of complex analysis, one finally obtains the charge density and charge fluctuations in terms of a frequency-dependent capacitance:

$$
\begin{aligned}
\left\langle\delta \sigma^{2}\right\rangle & =k_{B} T \sum_{n=-\infty}^{\infty} \lim _{\mathbf{r} \rightarrow \mathbf{r}^{\prime}} C\left(\mathbf{r}, \mathbf{r}^{\prime} ; i\left|\omega_{n}\right|\right) \\
& =k_{B} T \sum_{n=-\infty}^{\infty} \lim _{\mathbf{r} \rightarrow \mathbf{r}^{\prime}} \int \frac{d^{2} k}{(2 \pi)^{2}} C\left(\mathbf{k} ; i\left|\omega_{n}\right|\right) e^{i \mathbf{k} \cdot\left(\mathbf{r}-\mathbf{r}^{\prime}\right)},
\end{aligned}
$$

where $\mathbf{k}$ is the $2 \mathrm{D}$ wave vector. When the voltage fluctuations are distributed uniformly on the plates, the surface charge density fluctuations can be written in terms of the total charge fluctuations $\left\langle\delta q^{2}\right\rangle$ according to

$$
\left\langle\delta \sigma^{2}\right\rangle \rightarrow\left\langle\delta q^{2}\right\rangle=k_{B} T \sum_{n=-\infty}^{\infty} C\left(i\left|\omega_{n}\right|\right) .
$$

Using the electrostatic energy stored in the capacitor, $U=q^{2} / 2 C$, one finds the force per unit area between the capacitor plates as

$\bar{f}=-\frac{1}{2 A} \frac{\partial C^{-1}}{\partial z}\left\langle\delta q^{2}\right\rangle=-\frac{k_{B} T}{2 A} \frac{\partial C^{-1}}{\partial z} \sum_{n=-\infty}^{\infty} C\left(i\left|\omega_{n}\right|\right)$,

where $A$ is the area of the plate and $C(\omega)$ is the capacitance of the system. Note that here the frequency dependence comes from the response of the wire; therefore, in 
obtaining $\bar{f}$ from $U$ one does not retain the $\omega_{n}$ dependence in $\partial C^{-1} / \partial z$. In a more general case, however, to calculate the force, one needs to use $\bar{f}=-\frac{k_{B} T}{2 A} \sum_{n=-\infty}^{\infty} \frac{\partial C^{-1}\left(i\left|\omega_{n}\right|\right)}{\partial z} C\left(i\left|\omega_{n}\right|\right)$. We further elaborate on this point in what follows. Equation (7) constitutes a general expression for charge-induced fluctuation interactions. This Casimir-like effect is determined by the capacitance of the system. To understand the phenomenon further, the theory is applied to graphene systems with emphasis on thermal and quantum mechanical contributions.

The capacitance is obtained by considering that the charging of the capacitor leads to redistribution of carriers in space in order to minimize the electrostatic energy. One notes that the constant potential approximation is fulfilled since the size of the considered graphene systems is on the order of a micron, which is much larger than the screening length range of $1 \AA$ to a few $\mathrm{nm}$-s depending on the graphene carrier density [24]. Therefore, for classical systems the capacitance is determined by the geometry of the system via the geometrical capacitance $C_{0}$. For thin films, such as graphene and GNRs, the surface charges cannot completely shield the electrostatic field. This results in raising of the chemical potential to account for the increased density of states necessary for complete shielding [27]. The effect is associated with the quantum capacitance $C_{Q}$, and the total capacitance $C$ is determined by an addition of capacitors in series according to

$$
\frac{1}{C}=\frac{1}{C_{0}}+\frac{1}{A C_{Q}} .
$$

For the rectangular system in Fig. 1, the geometrical capacitance can be found [28] as

$$
C_{0}=\frac{A}{4}\left[2 z \arctan \left(\frac{w}{4 z}\right)+\frac{w}{4} \ln \left\{1+\left(\frac{4 z}{w}\right)^{2}\right\}\right]^{-1} .
$$

One notes that when the width of the ribbon $w$ is large, the parallel plate capacitance is recovered, $\bar{C}_{0}=A /(4 \pi z)$.
In addition, a quantum capacitance is not associated with the bottom plate since the thick metallic substrate screens the electrostatic field completely.

The quantum capacitance is determined by the particular material. For graphene it may be obtained via the random phase approximation $[14,25,29]$, as shown by several authors [30-32]. In the static limit $(\omega, \mathbf{k}) \rightarrow 0$, which is of relevance here, one finds

$C_{Q}=\frac{4 e^{2} k_{B} T}{\pi\left(\hbar v_{0}\right)^{2}} \ln \left[2 \cosh \left(\frac{\mu}{2 k_{B} T}\right)\right]=e^{2} \frac{\partial\left(n_{e}-n_{h}\right)}{\partial \mu}$,

where $n_{e}, n_{h}$ are the electron and hole concentrations, respectively. Also, $\mu$ is the chemical potential and $v_{0}=$ $10^{6} \mathrm{~m} / \mathrm{s}$ is the graphene carriers' velocity. The above expression reflects the fact that there is only a finite amount of charge available on the graphene sheet, $Q=e\left(n_{e}-n_{h}\right)$, due to the reduced dimensionality of graphene and its subsequent restriction on the density of states. Using Eqs. (8) and (10), an effective length parameter, associated with the partially screened field by the graphene, may be defined as $d^{*}=\left(\hbar v_{0}\right)^{2} /\left\{16 e^{2} k_{B} T \ln \left[2 \cosh \left(\mu / 2 k_{B} T\right)\right]\right\}$. At room temperature with no chemical potential, $d^{*} \approx 1 \mathrm{~nm}$.

The quantum capacitance for GNRs can be obtained in a similar manner. For narrow ribbons, however, the energy band structure is quantized due to the vanishing wave functions at the edges [33,34]. The effect of the energy quantization in the quantum capacitance can be seen in Fig. 2(a) for an armchair GNR with $w \approx 12 \mathrm{~nm}$. While the graphene $C_{Q}$ montonically increases as a function of $\mu$, the ribbon $C_{Q}$ experiences peaks [28,31]. This peak structure is much reduced if $T$ is raised to room temperature.

We note that the developed theory provides a general pathway to study a charge-induced fluctuations interaction in a capacitive system. This is essentially a Lifhsitz-like approach, which establishes a connection between the charge-induced interaction in Eq. (7) and the response of the system captured by the capacitance. The typical Casimir interaction, on the other hand, is related to the
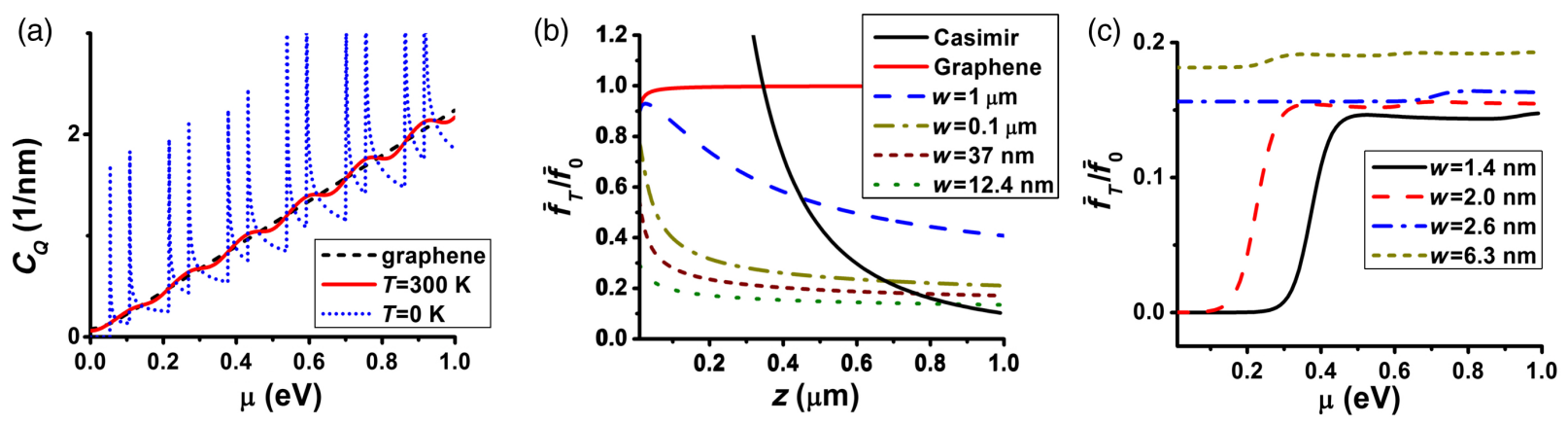

FIG. 2. (a) $C_{Q}$ as a function of chemical potential for graphene and a GNR with $w=12.4 \mathrm{~nm}$. (b) Charge fluctuations thermal force normalized by $\underline{f}_{0}=-k_{B} T /(2 A z)$. The area of all ribbon structures is $1 \mu \mathrm{m}^{2}$ and $\mu=0.1 \mathrm{eV}$. The graphene-metal Casimir force normalized by $\bar{f}_{0}$ at room temperature is also shown. The metal plasmon energy is taken as $\hbar \omega_{p}=9 \mathrm{eV}$. (c) The normalized charge fluctuations thermal force versus chemical potential for different GNRs at $T=300 \mathrm{~K}$. 
dielectric and magnetic response of the media. In general, the capacitance can be frequency dependent, as shown in Eqs. (1)-(6); therefore, we distinguish between thermal and quantum mechanical contributions in a similar manner as in the case of the Casimir force. The thermal $n=0$ Matsubara frequency is considered first. The capacitance in this case is determined by Eq. (8), containing the geometrical contribution and the static limit of the quantum capacitance [Eqs. (9) and (10)]. The force per unit area for a system with a wider GNR, in which the discrete band structure is not apparent (Fig. 1), is found analytically:

$$
\begin{aligned}
\bar{f}_{T}= & -\frac{4 k_{B} T}{A^{2}} C_{0}(z) \arctan \left(\frac{w}{4 z}\right) \\
& \times \frac{\frac{g e^{2} k_{B} T}{\pi\left(\hbar v_{0}\right)^{2}} \ln \left[2 \cosh \left(\frac{\mu}{2 k_{B} T}\right)\right]}{\frac{C_{0}(z)}{A}+\frac{g e^{2} k_{B} T}{\pi\left(\hbar v_{0}\right)^{2}} \ln \left[2 \cosh \left(\frac{\mu}{2 k_{B} T}\right)\right]} .
\end{aligned}
$$

For a graphene parallel plate capacitor, the geometrical capacitance is replaced by $\bar{C}_{0}=A /(4 \pi z)$ and $w \rightarrow \infty$ in $\bar{f}_{T}$. The above equation gives a unique expression for the thermal charge-induced fluctuation interaction involving graphene, which enables developing our further understanding of this type of force.

\section{RESULTS AND DISCUSSION}

The characteristic behavior of the thermal charge fluctuations interaction in terms of size of the system, separations, and quantum mechanical effects can now be examined. Figure 2(b) shows how the thermal force depends on the separation for both graphene and GNRs with different widths. For separations $z \gg d^{*}$, one finds that $C_{Q} \gg C_{0}$, and the thermal force is determined mainly by the geometrical capacitance. In the case of graphene, the force reduces to $\bar{f}_{0}=-k_{B} T /(2 A z)$. For separations $z \ll d^{*}, \bar{f}_{T}$ is determined mainly by the quantum capacitance. For narrower ribbons, the magnitude of the force is reduced, as seen in Fig. 2(b). While the effect of $C_{Q}$ for graphene is relatively small over a large part of the distance range $z$, its role for narrower ribbons can be much enhanced [Fig. 2(c)]. This feature, which reduces the magnitude of
$\bar{f}_{T}$, is attributed to the quantized band structure of the narrow ribbons.

It is important to compare how the typical Casimir force differs from the charge fluctuations induced interaction. The Casimir graphene-metallic substrate interaction has been calculated by the Lifshitz approach $[11,12]$, and it is also summarized in the Appendix. In Fig. 2(b) we show results for an infinite graphene sheet, described by the Dirac model, and a typical metal with a plasmon energy of $\hbar \omega_{p}=9 \mathrm{eV}$. Because of its $1 / z$ dependence, however, $\bar{f}_{T}$ is more important for larger $z$. It should be noted that the region where the size of the Casimir force is comparable to the charge fluctuation force is in that separation length where the thermal Casimir force is dominant (as opposed to the quantum mechanical contribution). This reduction in the distance scale where thermal effects take place, as compared to other systems, is due to the reduced dimensionality of graphene [19].

Figure 2(c) shows how the thermal charge fluctuations force evolves as a function of $\mu$ for GNRs with different widths. Because of the quantized electronic structure, $\mu$ affects the force much more for narrower ribbons. For wider ribbons, however, the interaction is hardly changed upon $\mu$.

The quantum mechanical contribution to the charge fluctuations force is closely related to the frequencydependent total capacitance. One must account for the particular mechanism for charging the capacitor, which happens via the connecting wire, as shown in Fig. 1(a). Taking a Drude model $[35,36]$ for the wire conductivity leads to a frequency-dependent total capacitance according to

$$
\frac{1}{C^{t}(\omega)}=\frac{1}{C_{0}}+\frac{1}{A C_{Q}}-i \omega R-\omega^{2} R \tau,
$$

where $R$ is the resistance of the wire and $\tau$ is the relaxation time [5,26,37]. Using Eqs. (7) and (12), the charge fluctuations force can now be calculated by taking into account all Matsubara frequencies. Results for the distance, $R$, and $\tau$ dependences of the force are shown in Fig. 3. Figure 3(a) shows the effect of the finite size of the ribbon; as the separation increases, the force is further reduced for all values of $R$. Also, it is found in Fig. 3(b) that smaller values of $R$ lead to an enhancement of the charge fluctuation force due to

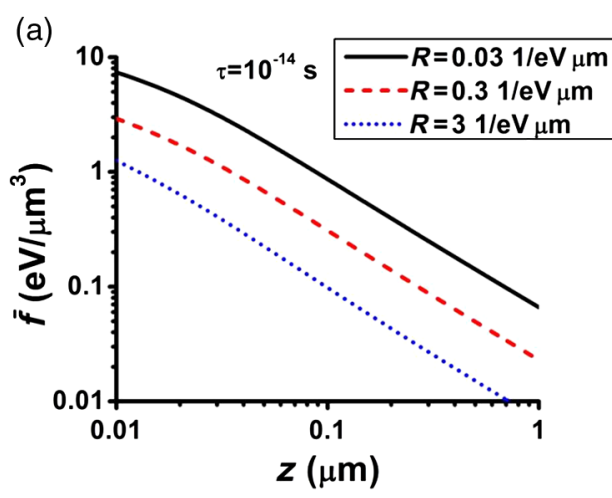

(b)

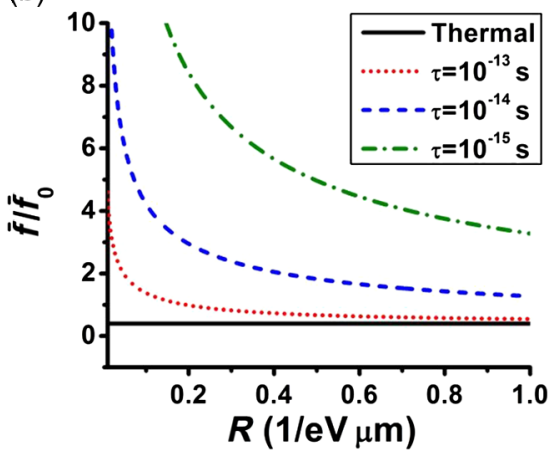

FIG. 3. (a) Charge fluctuations force versus distance between ribbon of width $0.1 \mu \mathrm{m}$ and area $1 \mu \mathrm{m}^{2}$ and a metal plate at room temperature, where $C^{t}(\omega)$ is used. Typical values for $\tau$ and $R$ are taken. (b) The normalized by $\bar{f}_{0}$ charge fluctuations force as a function of the wire resistance for different relaxation times. 
quantum mechanical effects while a larger resistance (compared to $\tau / C_{0}$ ) leads to thermal fluctuations dominating the interaction.

The quantum mechanical effects related to the mechanism of charging the capacitor can be analyzed further by considering the situation when $R C_{0}$ is small compared to $\tau$ and $C_{Q}$ can be neglected. The total capacitance in this case becomes $C^{t}(\omega) \approx C_{0} /\left(1-\omega^{2} R C_{0} \tau\right)$. From Eq. (7), the force is found as

$$
\bar{f}=-\frac{\hbar}{4 A} C_{0} \frac{\partial C_{0}^{-1}}{\partial z} \frac{1}{\sqrt{R C_{0} \tau}} \operatorname{coth}\left(\frac{\hbar}{2 k_{B} T \sqrt{R C_{0} \tau}}\right) .
$$

In the classical limit of large $T$ and large plate size compared to the separation $z$, one recovers $\bar{f}_{0}=-k_{B} T /(2 A z)$. In the quantum mechanical limit (with $z \ll \sqrt{A}$ ), where $\hbar / \sqrt{R C_{0} \tau} \gg k_{B} T$, the force for the graphene layer is found as

$$
\bar{f}=-\frac{\hbar}{2 A \sqrt{z}} \sqrt{\frac{\pi}{A R \tau}} .
$$

Equations (13) and (14) are instrumental in understanding Fig. 3 in a more transparent way. They clearly show how the role of $R$ affects the distance dependence of the interaction. For the quantum regime, which is characterized by smaller $R$, the dependence upon the separation goes as $1 / \sqrt{z}$. In the thermal regime, on the other hand, the force acquires an asymptotic distance behavior of $1 / z$.

Casimir and van der Waals forces are universal and of great importance in nature, especially when the scale goes down to micrometer distances and below. This type of interaction, induced by dipolar fluctuations, can be essential in determining the behavior of nanostructures and devices. Yet there are many other fluctuation-induced forces that become important at such small scales. Here, we consider charge-induced fluctuation forces in capacitive systems. The developed theory utilizes static and frequency-dependent capacitance, and it is applied in graphene-based structures. The total capacitance contains both a geometrical and a collective electronic components. Just like in the Casimir-van der Waals forces, we distinguish between thermal and quantum mechanical effects.

The origin of this induced interaction comes from fluctuating charges, which is a key difference when compared to the Casimir-van der Waals interaction that originates from fluctuating polarizations. This is important for understanding the $1 / z$ asymptotic distance dependence as compared to the $1 / z^{3}$ dependence of the thermal Casimir force. Similarly, the quantum mechanical regime of $1 / \sqrt{z}$ is of much longer range as compared to the metal-graphene quantum mechanical asymptotics $[11,12,14]$. It is also important to note that the geometrical capacitance takes into account the geometry of the system in a rather straightforward manner. This is unlike the Casimir force, where nontrivial geometry is usually difficult to describe theoretically. This is reflected in the fact that capacitance can be found for objects with nontrivial boundary conditions via electrostatic methods, while electromagnetic boundary conditions are not easily solved beyond highly symmetric extensions. Another distinct feature is the role of the size of the system. The charge fluctuations force is explicitly dependent on the area of the interacting objects, which is not the case for the Casimir-van der Waals force. The charge fluctuations interaction is $\sim 1 / A$ for the thermal and $\sim 1 / A^{3 / 2}$ for the quantum mechanical regimes.

We have demonstrated that in nanocapacitors a monopolar force arising from fluctuating charges transferred via a connecting wire can be made comparable or even larger in magnitude than the dipolar fluctuations forces at separations typical for the Casimir-van der Waals regimes. A key point here is the fact that the van der Waals-Casimir force in many nanostructures, including graphene systems, is much reduced as compared to bulk counterparts due to characteristic suppressions of the relevant plasma excitations $[19,20,22]$. Thus, controlling the properties of the graphene nanostructures and connecting wire provides an excellent opportunity for potentially observing the charge-induced fluctuation interaction. To better understand the quantitative side of this issue, we calculate that the charge-induced and Casimir interactions for the graphene system with $w=$ $1 \mu \mathrm{m}$ at $0.45 \mu \mathrm{m}$ are on the order of $26 \mathrm{~Pa}$ [see Fig. 2(b)]. As the separation is further increased to $0.6 \mu \mathrm{m}$, the chargeinduced fluctuation force becomes $17 \mathrm{~Pa}$, which is almost double the Casimir interaction of $10 \mathrm{~Pa}$ at that distance. We note that fluctuation-induced interactions of this magnitude can be accessed experimentally, as shown for the Casimir regime $[21,38]$. Therefore, we suggest that measurements in a nanocapacitor with and without the connecting wire might give means to distinguish between the typical Casimir and charge-induced Casimir-like interactions in an experimental setting. Challenges in terms of measurement accuracy might be overcome by exploiting the various factors, such as distance, area, and chemical potential to make a particular fluctuations force dominant. Nevertheless, how to engineer the specific setup with optimum conditions, where one can observe the different regimes for the charge-induced interaction, can be addressed by experimentalists in the future.

In summary, we argue that charge fluctuation forces are always present in the considered capacitorlike systems. They must be taken into account in conjunction with the standard Casimir interaction as the charge fluctuation force can be comparable or even bigger in magnitude. Charge fluctuation forces enable further probing of thermal and quantum mechanical effects due to fluctuation-induced phenomena in nanostructured materials such as graphene and GNRs. Finally, we point out that it is worthwhile to further pursue the effects of fluctuation forces due to not just electromagnetic fluctuations but also due to other thermodynamic parameters, for they will necessarily have an important effect in many nanostructures and devices. 


\section{ACKNOWLEDGMENTS}

We acknowledge financial support from the Department of Energy under Award No. DE-FG02-06ER46297. I. V. B. acknowledges support from the National Science Foundation under Award No. ECCS-1306871. R. P. would like to acknowledge the support of the U.S. Department of Energy, Office of Basic Energy Sciences, Division of Materials Sciences and Engineering under Award No. DE-SC0008176.

\section{APPENDIX: CASIMIR FORCE CALCULATIONS}

The Casimir force $f_{c}$ between a metallic substrate and graphene separated by a distance $z$ is presented here using the Lifshitz formalism [11,12,39],

$$
\begin{aligned}
f_{c}(z)= & -\frac{k_{B} T}{2 \pi} \sum_{n=-\infty}^{\infty} \int_{0}^{\infty} h\left(\omega_{n}\right) k_{\perp} d k_{\perp} \\
& \times\left\{\left[\frac{e^{2 h\left(\omega_{n}\right) z}}{\rho_{B}^{g r}\left(i\left|\omega_{n}\right|\right) \rho_{B}^{M}\left(i\left|\omega_{n}\right|\right)}-1\right]^{-1}\right. \\
& \left.+\left[\frac{e^{2 h\left(\omega_{n}\right) z}}{\rho_{E}^{g r}\left(i\left|\omega_{n}\right|\right) \rho_{E}^{M}\left(i\left|\omega_{n}\right|\right)}-1\right]^{-1}\right\},
\end{aligned}
$$

where $h\left(\omega_{n}\right)=\sqrt{\omega_{n}^{2} / c^{2}+k_{\perp}^{2}}, c$ is the speed of light in vacuo, and $\omega_{n}$ are the Matsubara frequencies. The reflection coefficients for the transverse magnetic mode are

$$
\begin{aligned}
& \rho_{B}^{\mathrm{gr}}(i \omega)=\frac{2 \pi \sigma(i \omega) h(\omega) / \omega}{1+2 \pi \sigma(i \omega) h(\omega) / \omega}, \\
& \rho_{B}^{M}(i \omega)=\frac{\epsilon(i \omega) h(\omega)-h_{M}(\omega)}{\epsilon(i \omega) h(\omega)+h_{M}(\omega)},
\end{aligned}
$$

where $\epsilon$ is the dielectric response of a typical metal and $h_{M}(\omega)=\sqrt{\epsilon(i \omega) \omega^{2} / c^{2}+k_{\perp}^{2}}$. The reflection coefficients for the transverse electric mode are

$$
\begin{aligned}
& \rho_{E}^{\mathrm{gr}}(i \omega)=-\frac{2 \pi \sigma(i \omega) \omega / h(\omega) c^{2}}{1+2 \pi \sigma(i \omega) \omega / h(\omega) c^{2}}, \\
& \rho_{E}^{M}(i \omega)=\frac{h(\omega)-h_{M}(\omega)}{h(\omega)+h_{M}(\omega)} .
\end{aligned}
$$

Using the two-band model for the conductivity of graphene [40] and a plasma model for a typical metal with the plasmon energy of $\sim 9 \mathrm{eV}$, one obtains the graphene-metal substrate force labeled as "Casimir" in Fig. 2(b).

[1] H. B. G. Casimir, Proc. K. Ned. Akad. Wet. 51, 793 (1948).

[2] I. E. Dzyaloshinskii, E. M. Lifshitz, and L. P. Pitaevskii, The General Theory of van der Waals Forces, Adv. Phys. 10, 165 (1961).
[3] D. Drosdoff and A. Widom, Fluid-Mechanical and Electrical Fluctuation Forces in Colloids, Phys. Rev. E 73, 051402 (2006).

[4] A. Einstein, Über die Gültigkeitsgrenze des Satzes vom thermodynamischen Gleichgewicht und über die Möglichkeit einer neuen Bestimmung der Elementarquanta, Ann. Phys. (Berlin) 327, 569 (1907).

[5] J. B. Johnson, Thermal Agitation of Electricity in Conductors, Phys. Rev. 32, 97 (1928).

[6] H. Nyquist, Thermal Agitation of Electrical Charge in Conductors, Phys. Rev. 32, 110 (1928).

[7] M. T. Homer Reid, A. W. Rodriguez, and S. G. Johnson, Fluctuation-Induced Phenomena in Nanoscale Systems: Harnessing the Power of Noise, Proc. IEEE 101, 531 (2013).

[8] J. Kirkwood and J. B. Shumaker, Forces between Protein Molecules in Solution Arising from Fluctuations in Proton Charge and Configuration, Proc. Natl. Acad. Sci. U.S.A. 38, 863 (1952).

[9] R. Podgornik, Electrostatic Correlation Forces between Surfaces with Surface Specific Ionic Interactions, J. Chem. Phys. 91, 5840 (1989).

[10] A. Naji, D. S. Dean, J. Sarabadani, R. R. Horgan, and R. Podgornik, Fluctuation-Induced Interaction between Randomly Charged Dielectrics, Phys. Rev. Lett. 104, 060601 (2010).

[11] G. L. Klimchitskaya, V. M. Mostepanenko, and B. E. Sernelius, Two Approaches for Describing the Casimir Interaction in Graphene: Density-Density Correlation Function versus Polarization Tensor, Phys. Rev. B 89, 125407 (2014).

[12] M. Bordag, G. L. Klimchitskaya, and V. M. Mostepanenko, Thermal Casimir Effect in the Interaction of Graphene with Dielectrics and Metals, Phys. Rev. B 86, 165429 (2012).

[13] V. V. Gobre and A. Tkatchenko, Scaling Laws for the van der Waals Interactions in Nanostructured Materials, Nat. Commun. 4, 2341 (2013).

[14] J. F. Dobson, A. White, and A. Rubio, Asymptotics of the Dispersion Interaction: Analytical Benchmarks for the van der Waals Energy Functionals, Phys. Rev. Lett. 96, 073201 (2006).

[15] J. F. Dobson, T. Gould, and G. Vignale, How Many-Body Effects Modify the van der Waals Interaction between Graphene Sheets, Phys. Rev. X 4, 021040 (2014).

[16] G. L. Yu, R. Jalil, B. Belle, A. S. Mayorov, S. Alexander, P. Blake, F. Schedin, S. V. Morozov, L. A. Ponomarenko, F. Chiappini, S. Wiedmann, U. Zeitler, M. I. Katsnelson, A. K. Geim, K. S. Novoselov, and D. C. Elias, Interaction Phenomena in Graphene Seen through Quantum Capacitance, Proc. Natl. Acad. Sci. U.S.A. 110, 3282 (2013).

[17] S. Ilani, L. A. K. Donev, M. Kindermann, and P. L. McEuen, Measurement of the Quantum Capacitance of Interacting Electrons in Carbon Nanotubes, Nat. Phys. 2, 687 (2006).

[18] S. Luryi, Quantum Capacitance Devices, Appl. Phys. Lett. 52, 501 (1988).

[19] G. Gomez-Santos, Thermal van der Waals Interaction between Graphene Sheets, Phys. Rev. B 80, 245424 (2009).

[20] B. E. Sernelius and P. Bjork, Interactions Energy for a Pair of Quantum Wells, Phys. Rev. B 57, 6592 (1998). 
[21] F. Intravaia, S. Koev, I. W. Jung, A. A. Talin, P. S. Davis, R. S. Decca, V. A. Aksyuk, D. A. R. Dalvit, and D. Lopez, Strong Casimir Force Reduction through Metallic Surface Nanostructuring, Nat. Commun. 4, 2515 (2013).

[22] D. Drosdoff and L. M. Woods, Quantum and Thermal Dispersion Forces: Application to Graphene Nanoribbons, Phys. Rev. Lett. 112, 025501 (2014).

[23] R. Kubo, Statistical-Mechanical Theory of Irreversible Processes: I. General Theory and Simple Applications to Magnetic and Conduction Problems, J. Phys. Soc. Jpn., 12, 570 (1957).

[24] The in-plane screening length $a_{s}=1 / q_{s}$ in graphene can be estimated using Thomas-Fermi theory [25,26]. Using the two band Dirac-like model, the dielectric response function is found as $\epsilon=1+q_{s} / q$, where $q$ is the $2 \mathrm{D}$ wave vector and $q_{s}=\left(8 \alpha c k_{B} T / \hbar v_{0}^{2}\right) \ln \left[2 \cosh \left(\mu / 2 k_{B} T\right)\right]$.

[25] E. H. Hwang and S. Das Sarma, Dielectric Function, Screening, and Plasmons, in Two-Dimensional Graphene, Phys. Rev. B 75, 205418 (2007).

[26] T. Ando, Screening effect and Impurity Scattering in Monolayer Graphene, J. Phys. Soc. Jpn. 75, 074716 (2006).

[27] T. Kopp and J. Mannhart, Calculation of the Capacitances of Conductors: Perspectives for the Optimization of Electronic Devices, J. Appl. Phys. 106, 064504 (2009).

[28] A. A. Shylau, J. W. Klos, and I. V. Zozoulenko, Capacitance of Graphene Nanoribbons, Phys. Rev. B 80, 205402 (2009).

[29] A. H. Castro Neto, F. Guinea, N. M. R. Peres, K. S. Novoselov, and A. K. Geim, The Electronic Properties of Graphene, Rev. Mod. Phys. 81, 109 (2009).

[30] K. Lam and J. Guo, Frequency-Dependent Quantum Capacitance and Plasma Wave in Monolayer Transition
Metal Dichalcogenides, Appl. Phys. Lett. 104, 103111 (2014).

[31] T. Fang, A. Konar, H. Xing, and D. Jena, Carrier Statistics and Quantum Capacitance of Graphene Sheets and Ribbons, Appl. Phys. Lett. 91, 092109 (2007).

[32] J. Xia, F. Chen, J. Li, and N. Tao, Measurement of the Quantum Capacitance of Graphene, Nat. Nanotechnol. 4, 505 (2009).

[33] L. Brey and H. A. Fertig, Elementary Electronic Excitations in Graphene Nanoribbons, Phys. Rev. B 75, 125434 (2007).

[34] L. Brey and H. A. Fertig, Electronic States of Graphene Nanoribbons Studied with the Dirac Equation, Phys. Rev. B 73, 235411 (2006).

[35] P. Drude, Zur Elektronentheorie der Metalle, Ann. Phys. (Berlin) 306, 566 (1900).

[36] L. Solymar and D. Walsh, Lectures on the Electrical Properties of Materials, (Oxford University Press, Belfast, 1970).

[37] D.-Y. Jeon, K. J. Lee, M. Kim, D. C. Kim, H.-J. Chung, Y.-S. Woo, and S. Seo, Radio-Frequency Electrical Characteristics of Single Layer Graphene, Jpn. J. Appl. Phys. 48, 091601 (2009).

[38] A. A. Banishev, H. Wen, J. Xu, R. K. Kawakami, G. L. Klimchitskaya, V. M. Mostepanenko, and U. Mohideen, Measuring the Casimir Force from Graphene on a $\mathrm{SiO}_{2}$ Substrate, Phys. Rev. B 87, 205433 (2013).

[39] D. Drosdoff and L. M. Woods, Casimir Forces and Graphene Sheets, Phys. Rev. B 82, 155459 (2010).

[40] L. A. Falkovsky and A. A. Varlamov, Space-Time Dispersion of Graphene Conductivity, Eur. Phys. J. B 56, 281 (2007). 